\title{
Modulating the Biomechanical Properties of Engineered Connective Tissues by Chitosan-Coated Multiwall Carbon Nanotubes
}

This article was published in the following Dove Press journal: International Journal of Nanomedicine

\author{
Naim Kittana (D) \\ Mohyeddin Assali (D) ${ }^{2}$ \\ Wolfram-Hubertus \\ Zimmermann (iD) 3,4 \\ Norman Liaw (iD) 3,4 \\ Gabriela Leao Santos (iD) 3,4 \\ Abdul Rehman ${ }^{3,4}$ \\ Susanne Lutz iD 3,4 \\ 'Department of Biomedical Sciences, \\ Faculty of Medicine \& Health Sciences, \\ An-Najah National University, Nablus, \\ Palestine; ${ }^{2}$ Department of Pharmacy, \\ Faculty of Medicine \& Health Sciences, \\ An-Najah National University, Nablus, \\ Palestine; ${ }^{3}$ Institute of Pharmacology and \\ Toxicology, University Medical Center \\ Göttingen, Göttingen, Germany; ${ }^{4}$ DZHK \\ (German Center for Cardiovascular \\ Research) Partner Site Göttingen, \\ Göttingen, Germany
}

Background: Under certain conditions, the physiological repair of connective tissues might fail to restore the original structure and function. Optimized engineered connective tissues (ECTs) with biophysical properties adapted to the target tissue could be used as a substitution therapy. This study aimed to investigate the effect of ECT enforcement by a complex of multiwall carbon nanotubes with chitosan (C-MWCNT) to meet in vivo demands.

Materials and Methods: ECTs were constructed from human foreskin fibroblasts (HFF-1) in collagen type I and enriched with the three different percentages $0.025,0.05$ and $0.1 \%$ of C-MWCNT. Characterization of the physical properties was performed by biomechanical studies using unidirectional strain.

Results: Supplementation with $0.025 \%$ C-MWCNT moderately increased the tissue stiffness, reflected by Young's modulus, compared to tissues without C-MWCNT. Supplementation of ECTs with $0.1 \%$ C-MWCNT reduced tissue contraction and increased the elasticity and the extensibility, reflected by the yield point and ultimate strain, respectively. Consequently, the ECTs with $0.1 \%$ C-MWCNT showed a higher resilience and toughness as control tissues. Fluorescence tissue imaging demonstrated the longitudinal alignment of all cells independent of the condition.

Conclusion: Supplementation with C-MWCNT can enhance the biophysical properties of ECTs, which could be advantageous for applications in connective tissue repair.

Keywords: engineered connective tissue, multiwall carbon nanotubes, chitosan, mechanical properties, collagen-based tissue scaffold

\section{Introduction}

Regenerative medicine is gaining momentum in various applications. Repairing and replacing damaged tissues and organs by tissue-engineered surrogates is a promising discipline in regenerative medicine, with compelling evidence for applications to accelerate healing processes and to restore the tissue and organ functions. ${ }^{1-4}$

Connective tissue is the most abundant tissue type in the human body. Its major functions include the support, protection, and connection of other tissues ${ }^{5,6}$ and it forms various components of the body like tendons, ligaments, the dermis layer of the skin, and the cardiac skeleton. Dependent on the function, the composition of connective tissue varies. Besides the ECM producing cells, collagens are the most abundant constituents together with other proteins like elastin, fibrillin, fibronectin, and laminin. ${ }^{6-8}$

Connective tissues are frequently prone to serious injuries, where the physiological regeneration might fail to restore the original structure and function.
Correspondence: Naim Kittana

Department of Biomedical Sciences,

Faculty of Medicine and Health Sciences,

An Najah National University, Akademia

Street, Nablus, Palestine

$\mathrm{Tel}+970$ (9) $2345 \mathrm{II} 3$

Fax +970 (9) 2345982

Email naim.kittana@najah.edu 
Therefore, many research groups are trying to develop new approaches in tissue engineering for the aim of generating engineered connective tissues (ECTs) ${ }^{3,9}$ with properties that specifically meet the anatomical and physiological requirements of the targeted connective tissue type. However, there is a lack of informative data on their mechanical characteristics and the ways to further optimize their physiological performance.

Numerous macromolecules were studied for potential use as scaffolds for tissue engineering; chitosan was among the materials that showed promising results. It is a natural non-toxic polymer, which can be used for the fabrication of biodegradable 3D scaffolds in a variety of geometries. ${ }^{10}$ Carbon nanotubes (CNTs) are also interesting materials for tissue engineering due to their unique electrical and mechanical properties. Their electrical conductivity can reach up to 1000 times greater than copper wires, ${ }^{11}$ while their tensile strength can be up to 63 gigapascal $(\mathrm{GPa})$, which is around 50 times greater than that of steel. ${ }^{12}$ At the same time, their elastic modulus can reach between 1 to 1.8 terapascal (Tpa). ${ }^{13}$ CNTs are made of sheets of six-membered carbon atom rings (graphite) that wrap into cylindrical tubes ${ }^{14,15}$ with a diameter in the nanometer scale and a length in the micrometer scale. ${ }^{16,17}$ Depending on the number of the graphene layers, CNTs can be classified into single-walled or multi-walled (MWCNT) ${ }^{18}$ The organization of CNTs in the form of a three-dimensional (3D) configuration is necessary for the utilization of the unique CNTs' physicochemical properties in the construction of tissues. It has been shown that MWCNT cross-linked into a porous 3D scaffold formed cytocompatible surfaces for the growth of osteoblast precursor cells. ${ }^{19}$ A study published by our group investigated the effects of chitosan-coated MWCNT (C-MWCNT) on the behavior of dermal fibroblasts in ECTs. The study demonstrated that the fibroblasts were able to organize collagen enriched with C-MWCNT and thereby they formed connective tissues. Besides, a 2D culture of fibroblasts with CNTs coated with chitosan showed that the presence of CNT increased the deposition of collagen by fibroblasts. Additionally, we demonstrated that the topical application of a chitosan hydrogel enriched with CNT in a mouse wound model accelerated wound closure and enhanced fibrosis of the dermis and epithelialization of the epidermis layer. ${ }^{20}$ The utilization of modified MWCNT in the generation of scaffolds with enhanced mechanical and biological properties was reported before by Zhang and colleagues, who showed that these scaffolds were compatible with bone mesenchymal stem cells growth and differentiation, and that they also effectively enhanced bone regeneration in vivo. ${ }^{21}$ Furthermore, other modified carbon allotropes, like graphene oxide, incorporated in composite scaffolds of collagen, chitosan, and alginate also demonstrated improvements in their mechanical properties and could serve as promising scaffold for engineered bone tissues. ${ }^{22}$ Another interesting paper by Dai and colleagues reported a description for the generation of $3 \mathrm{D}$ high-porosity chitosan/honeycomb porous carbon/hydroxyapatite scaffold that could enhance the differentiation of bone mesenchymal stem cells into the osteogenesis direction. Besides the reported in vivo data demonstrated an enhanced bone regeneration. ${ }^{23}$

In this project, collagen-based ECTs were generated containing primary human foreskin fibroblasts. These ECTs were enriched with different concentrations of C-MWCNT to enhance their mechanical properties.

\section{Materials and Methods}

Primary human foreskin fibroblasts-1 (HFF-1), cat. SCRC1041, LOT 63,229,645 were obtained from the American Type Culture Collection [ATCC] (Manassas, VA, USA). Acid-soluble bovine collagen $(\sim 6 \mathrm{mg} / \mathrm{mL})$ was purchased from Collagen Solutions (Glasgow, UK). DMEM powder, DMEM high glucose GlutaMAX, fetal bovine serum (FBS) and all other cell culture reagents were obtained from Life Technologies (Carlsbad, CA, USA). Multiwall carbon nanotubes (MWCNT) were obtained from Carbon Solutions (Riverside, CA, USA). Roti-Histofix and RotBlock were obtained from Carl Roth (Karlsruhe, Germany). DAPI, chitosan, acetic acid, and tetramethylrhodamine (TRITC)-phalloidin were purchased from Sigma-Aldrich (St Louis, MO, USA). Sterile water (Aqua) was purchased from Braun (Kronberg im Taunus, Germany). Zeiss Lumar.V12 stereomicroscope (München, Germany) was used for macroscopic imaging of ECTs. VT1000S Vibratome ${ }^{\circledR}$ from Leica (Wetzlar, Germany) was used to section the tissues. An Olympus IX 81 inverted fluorescence microscope (Hamburg, Germany) was used for tissue microscopic examination. RSA-G2 solids analyzer from TA Instruments (New Castle, DE, USA) was used for the studies on the biomechanical properties of the tissues. 


\section{Preparation of Chitosan-MWCNT Complex (C-MWCNT)}

Chitosan solution with a concentration of $0.5 \%(\mathrm{w} / \mathrm{v})$ was prepared in $0.5 \%$ acetic acid $(\mathrm{v} / \mathrm{v})$ aqueous solution. MWCNT was added to the acidic chitosan solution to obtain a mixture of $4 \%(\mathrm{w} / \mathrm{v})$. Then, the mixture was sonicated for $2 \mathrm{~h}$ and autoclaved thereafter.

\section{Generation and Processing of Engineered Connective Tissues (ECTs)}

HFF-1 cells were maintained and propagated in a growth medium composed of high glucose DMEM containing $15 \% \mathrm{FBS}$ and $1 \%$ penicillin/streptomycin. The ECTs were generated according to a method described in recent publications by our lab, ${ }^{20,24}$ which was a modified method from Tiburcy and colleagues. ${ }^{25}$ Briefly, DMEM powder was used to prepare 10x DMEM, which was further used to prepare 2x DMEM by dilution in water and the addition of FBS up to $20 \%$. C-MWCNT solution was mixed with 2x DMEM before it was homogeneously mixed with collagen $(0.3 \mathrm{mg}$ per tissue) and neutralized by $0.1 \mathrm{~N} \mathrm{NaOH}$. Afterwards, the HFF-1 cell suspension was immediately added, so that each $200 \mu \mathrm{L}$-sized ECT contained 750,000 cells. The mixture was homogenized by repeated gentle pipetting. Up to this step, all processes were performed on ice. Then, $200 \mu \mathrm{L}$ of the mixtures were cast in a 48 -well mold plate containing two flexible poles (Myriamed, Göttingen, Germany). The plate was kept in a cell culture incubator for $1 \mathrm{~h}$ at $37^{\circ} \mathrm{C}$ before the HFF-1 growth medium was added. Tissue condensation occurred within $24 \mathrm{~h}$ and the culture of the ECTs was carried out for 5 days in a standard cell culture incubator $\left(37^{\circ} \mathrm{C}\right.$, $5 \% \mathrm{CO}_{2}$ and $99 \%$ humidity). The medium was changed every other day.

\section{Studying the Biomechanical Properties of ECT}

On the fifth day, ECT contraction was determined by calculating the percent change in the distance between the two flexible poles. Therefore, the ECTs were imaged with a Lumar.V12 stereomicroscope (Zeiss) and the distances were measured with ImageJ. In addition, the stereomicroscope was used to take calibrated ECT images from the top and side. These images were also analyzed by ImageJ software, and the resulting dimensional data were fed in Microsoft Excel 2007 to calculate the cross-sectional area (CSA). Next, each ECT was placed on two opposite hooks attached to the unidirectional strain rheometer set-up in a thermostatted organ bath $\left(37^{\circ} \mathrm{C}\right)$ containing PBS. The ECTs were elongated with a strain rate of $0.03 \mathrm{~mm} / \mathrm{s}$ along their longitudinal axis and tensile forces with a sensitivity of 0.1 millinewton $(\mathrm{mN})$ were recorded until tissue rupture. The stress was calculated for each point based on the measured force and the CSA of each ECT:

Stress $=$ Tensile force/cross-sectional area

While the strain was calculated as relative deformation of the tissue:

Strain $=($ New length - Starting length $) /$ Starting length .

The obtained stress-strain curves were used to calculate the Young's modulus, which reflects the stiffness of the tissues. Therefore, the stress-strain curves were zeroed in $\mathrm{X}$ - and Y-directions, and the slope of the initial linear elastic phase was determined by linear regression. The yield point stress, the proportional limit, and the yield point strain were determined as the points where the change in stress is no longer linear with the change in strain. The maximum stress was determined as the peak recorded stress. The ultimate tensile stress and strain were determined as the stress and strain required for an ECT to break. The total area under the stress-strain curves and the area under the elastic region of the curves were calculated by GraphPad Prism version 7 software to determine the toughness and the resilience of the ECT, respectively (Figure 1).

\section{Tissue Processing and Fluorescence Imaging}

For histology, the ECTs were fixed in Roti-Histofix overnight, then washed three times with PBS and once with $70 \%$ ethanol before they were embedded in $2 \%$ agarose. The tissues were sectioned in $100 \mu \mathrm{m}$ slices with a VT1000S vibratome (Leica Biosystems, Wetzlar, Germany). The tissue slices were permeabilized with $0.05 \%$ Triton X-100 in PBS for $10 \mathrm{~min}$, blocked for $1 \mathrm{~h}$ in Roti-Block, and then stained overnight with $0.5 \mu \mathrm{g} / \mathrm{mL}$ TRITC-phalloidin and $0.5 \mu \mathrm{g} / \mathrm{mL}$ DAPI in PBS in the dark at $4^{\circ} \mathrm{C}$. Imaging was performed with an Olympus IX 81 inverted fluorescence microscope. 


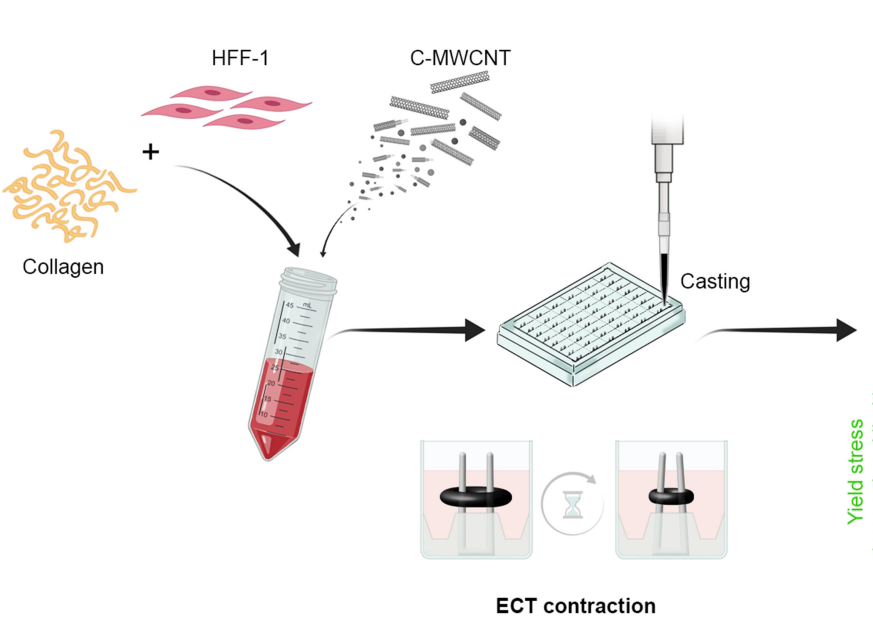

Specification of composition

- Defined composition

- Flexibility in the used components

- Gain- and loss-of function analyses easy to achieve

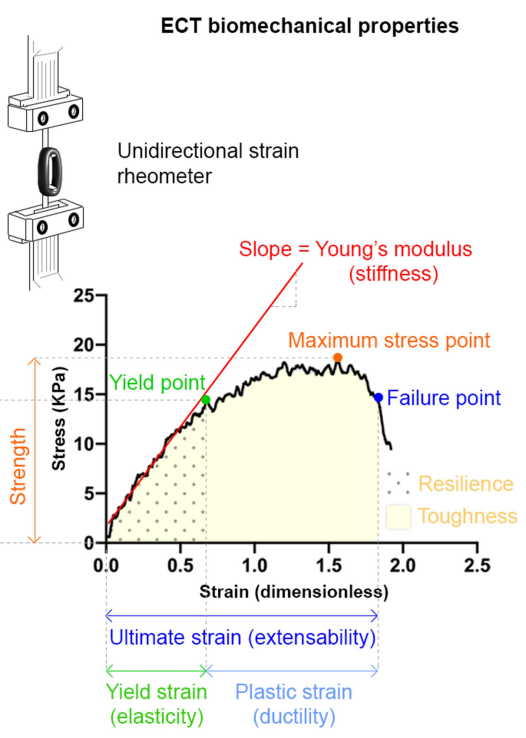

Biomechanical measurements

- Ring-shape allows for readily study mechanics, structure and function

- No artificial breaking points due to clamping the tissue

- Long-term morphology and contraction analysis under sterile conditions possible

- Possibility of tuning the stiffness of the retaining poles

- Ring-shaped molds results in anisotropic tissues

- Applicability to drug screening.

Figure I Schematic representation of the used ECT model. HHF-I were mixed with bovine collagen and different percentages of C-MWCNT to generate ECTs in a 48-well mold plate. Each mold was equipped with two flexible poles with a spring constant of $1.5 \mathrm{mN} / \mathrm{mm}$. At the end of the 5 days culture period, tissue contraction was analyzed based on the decline in pole distance. Then, the ECTs were subjected to ultimate uniaxial tensile measurements and the obtained stress-strain curves were used for the analysis of different biomechanical parameters. The herein presented parameters together with their functional equivalents are depicted in the representative stress-strain curve. The most important advantages of the used methods are listed underneath the scheme. Data from references. ${ }^{42-52}$

\section{Statistics}

Data are presented as arithmetic mean+standard error of the mean (SEM). The sample number is indicated as n. For statistical testing a one-way ANOVA with an appropriate post hoc test was applied.

\section{Results and Discussion}

Healing of deep wounds is a major complication in some diseases. Among the various processes required for the healing of deep wounds, fibroblast activation is an important step. Activated fibroblasts proliferate and migrate more intensively than quiescent fibroblasts and further transdifferentiate into myofibroblasts, which deposit ECM components and contract the wound edges. $^{26,27}$ These processes are often disturbed in chronic wounds, like in Diabetes mellitus, where the occurrence of myofibroblasts is delayed or missing. ${ }^{28,29}$ Therefore, the generation of collagen-based tissue substitutes might be an attractive approach for promoting the healing process, because in some tissues the grafted 
collagen-based tissues can act as a sacrificial substrate for enhanced proteolytic processes via matrix metalloproteinases. This may negatively impair the balance between collagen deposition and turnover, thus giving a better opportunity for the newly deposited collagen to accumulate and fill in the gap. ${ }^{30}$ Besides, the breakdown products of collagen can act as chemoattractants for infiltrating and migrating cells from the surroundings towards the provisional matrix, which can effectively facilitate the healing of the tissues. ${ }^{31}$ In general, we believe that such engineered connective tissues should have certain physical and biological properties. First, the use of cells, which display a more pronounced myofibroblastic phenotype would be preferable with respect to their ability to form compact tissues and to secrete ECM proteins necessary for wound healing. Second, the grafted tissues should not contract. Third, the tissues should be resistant to pulling and compression forces that enable them to withstand the relatively high physical stressors in the engraftment region. However, these three exigencies are hardly achievable with collagen-based tissues as fibroblast on the one hand do not show a high secretory activity and myofibroblasts, on the other hand, tend to form contracted, stiff and brittle tissues, which cannot resist well the high pulling forces. ${ }^{32,33}$ In our opinion, an additional scaffold component is necessary to fulfill the three requirements. In that respect, MWCNT can be an interesting material based to their unique physical properties. They especially stand out due to their high tensile strength and elastic modulus. ${ }^{12,13}$ However, pristine MWCNT (without any modifications) are very hydrophobic, thus they tend to aggregate and precipitate in aqueous environments. Besides, they are not biocompatible. Therefore, to utilize MWCNT in the construction of engineered tissues, they have to be coated with a hydrophilic polymer, such as chitosan, so that they become biocompatible and dispersible in hydrophilic environments. ${ }^{10,34-36}$ In addition, chitosan offers several biological advantages, eg it acts as a substrate for cell adhesion and has anti-microbial activity. ${ }^{37-41}$

In this project, we generated ECTs composed of primary HFF-1 and bovine collagen, which were supplemented with different percentages of C-MWCNT. We analyzed tissue compaction and contraction and performed an extensive analysis of the biomechanical tissue properties (Figure 1). The main advantages of the used methods are summarized in Figure 1. Additionally, we sectioned the tissues to investigate the morphology of the embedded cells.

\section{C-MWCNT Impairs Tissue Contraction of ECTs}

As describe above, the ECTs without or with C-MWCNT were cultured for 5 days in the 48-well mold plates. During this time the embedded fibroblasts contracted the ring-shaped, collagen-based ECTs, thus bringing the pair of elastic poles closer to each other (Figure 2A). The degree of tissue contraction was assessed by determining the percent change of the distance between the two flexible poles of each mold. ECTs without C-MWCNT contracted the poles by around $20 \%$, which was not affected by the addition of $0.025 \%$ C-MWCNT. However, in the presence of $0.1 \%$ C-MWCNT, ECT contraction was significantly impaired (Figure 2B).

The, the ECTs were released from the casting molds and imaged to obtain the CSA. No differences in CSA were observed in the investigated groups (Figure 3).

The results obtained for the ECTs with $0.1 \%$ C-MWCNT were particularly distinct. In these tissues, the ability of the embedded cells to contract the matrix was hampered, which in our belief was not due to a failure in fibroblast functionality, as the cell-driven tissue compaction was unaffected as demonstrated by the similar CSA of all tissue types. Moreover, chitosan-covered carbon nanotubes were demonstrated to be highly biocompatible in engineered tissues containing fibroblasts, epithelial cells, neuronal cells and cardiomyocytes and thus cytotoxicity could be excluded as an explanation. ${ }^{53-56} \mathrm{We}$ hypothesize that the observed impairment in tissue contraction is based on sterical hindrance exerted by the large amounts of the C-MWCNT embedded in the tissues. In line, similar inhibitory effects on tissue contraction, for both carbon nanotubes and chitosan, were previously reported. ${ }^{56}$

\section{C-MWCNT Modulated the Physical Characteristics of ECTs}

The ECTs were subjected to uniaxial tensile testing for stress-strain analysis. In general, the obtained curves demonstrated that the ECTs showed an initial linear elastic phase followed by a plastic phase in which the stress and strain values were no longer proportional to each other 
A
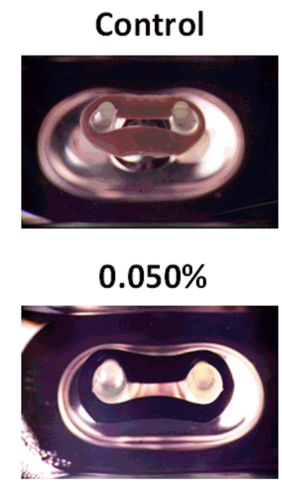

\section{$0.025 \%$}

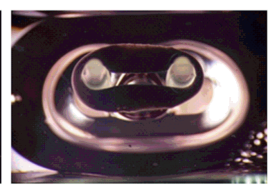

$0.100 \%$

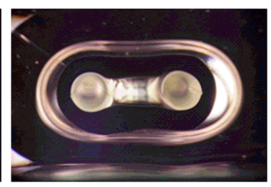

B

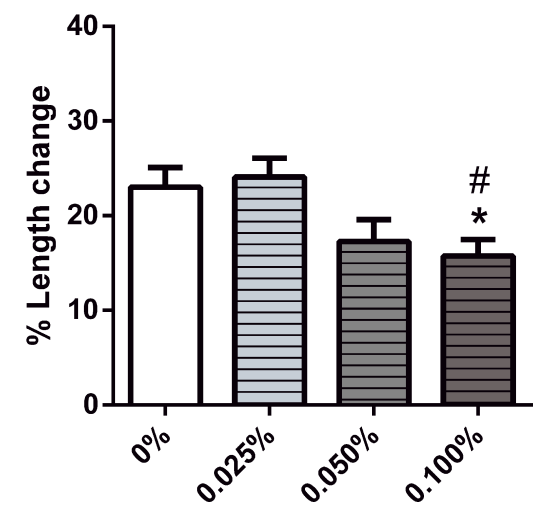

Figure 2 Impact of C-MWCNT on ECT contraction. ECTs were constructed in a 48-well mold plate. (A) Representative ECTs with different concentrations of C-MWCNT in the casting molds are shown. (B) Analysis of the ECT contraction is given; the presence of $0.1 \%$ C-MWCNT significantly reduced the contraction of ECTs. * $p<0.05$ compared to control and ${ }^{\#} p<0.05$ compared to 0.025 , as assessed by a one-way ANOVA with Tukey's post hoc testing for multiple comparisons; $n=12-13$ ECTs/group.

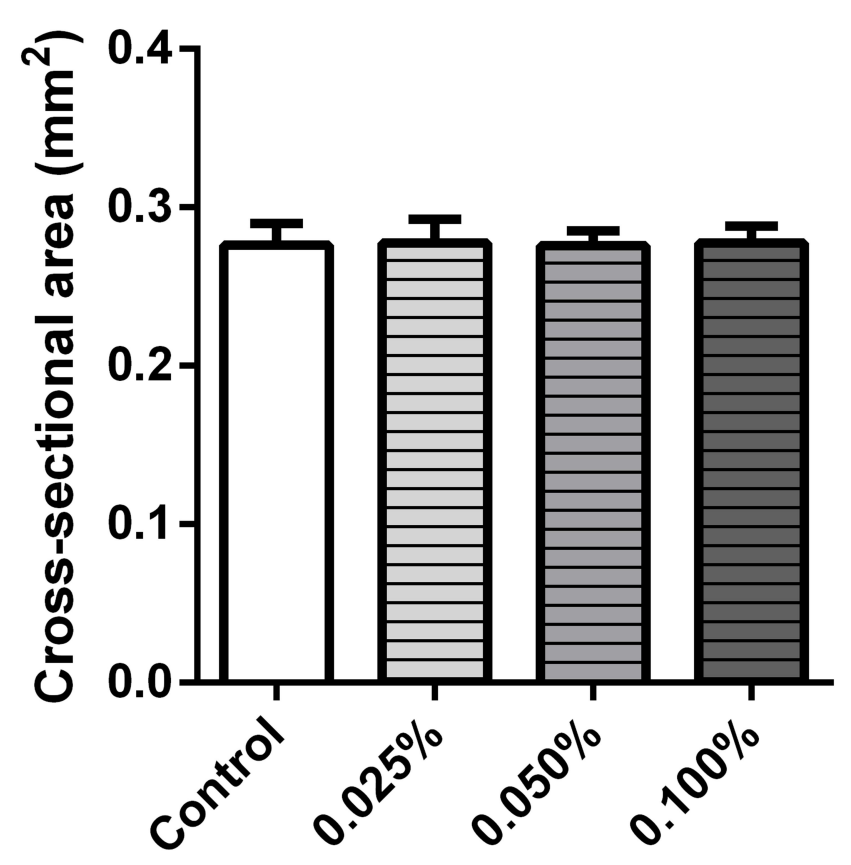

Figure 3 Effect of C-MWCNT on the cross-sectional area of the ECT. The incorporation of C-MWCNT showed no significant effect on the cross-sectional area of the ECTs in comparison with the control, $n=8-9 \mathrm{ECT}$ /group.

(Figure 4A). Several parameters were retrieved from the curves and are presented, or were used to calculate the amount of absorbed energy.

First, the data demonstrates that $0.025 \%$ C-MWCNT moderately, but significantly increased the stiffness of the ECTs given by the increase in Young's modulus, which corresponds to the slope of the elastic phase. Higher contents of C-MWCNT were without influence on tissue stiffness compared to control (Figure 4B). Second, the addition of $0.1 \%$ C-MWCNT, but not of lower percentages, profoundly influenced the yield point of the ECTs, which marks the transition from the elastic to the plastic phase. The proportional limit, equaling the stress at yield point, and the yield point strain were both increased compared to control (Figure 4C and D). Consequently, the resilience, calculated as the area under the curve (AUC) of the elastic phase, was higher in the presence of $0.1 \%$ C-MWCNT (Figure 4E). This indicated that these tissues were able to absorb more energy within the elastic phase mainly based on an elastic phase elongation and not due to an increase in Young's modulus. In line with the elongated elastic phase, tissues containing $0.1 \%$ C-MWCNT showed higher maximum tensile strength (Figure 4F). Third, tissue ductility was also increased in the presence of $0.1 \%$ C-MWCNT as demonstrated by the increased plastic strain, which represents the delta between the yield point strain and the ultimate strain (Figure 4G). The increases in the elastic and plastic strains resulted consequently in an elongated ultimate strain, meaning enhanced tissue extensibility (Figure 4H). Finally, curve elongation increased the area under the total curve indicating enhanced tissue toughness (Figure 4I).

Taken together, our data demonstrate that the effects of the supplementation of ECTs with C-MWCNT on the biomechanical tissue properties critically depend on the added concentrations. We show that ECTs containing $0.025 \%$ C-MWCNT displayed a moderate increase in tissue stiffness. This increase was accompanied by a likewise moderate, although not significant decline in the yield point strain and resilience. This indicates that the tissues were not only stiffer, but also less elastic. Such an inverse relationship between increased stress 
A

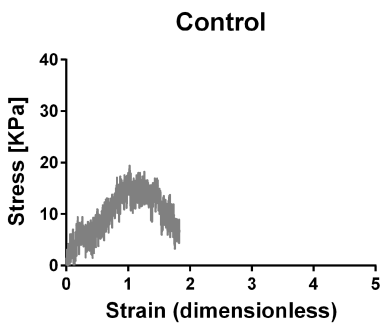

B

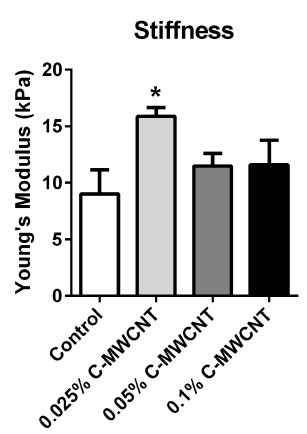

F

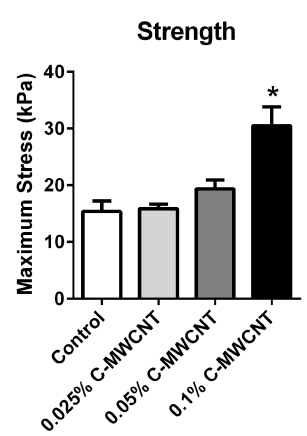

$0.025 \%$ C-MWCNT

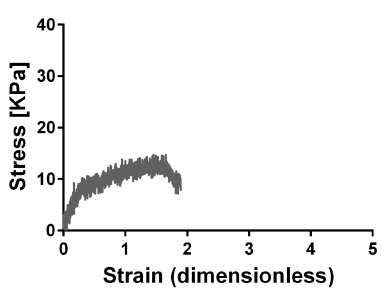

C

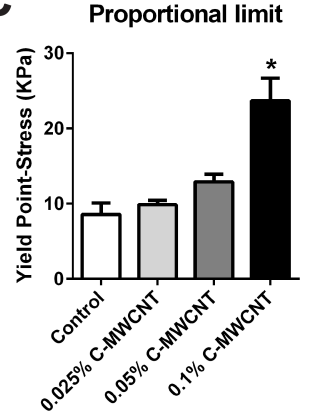

G

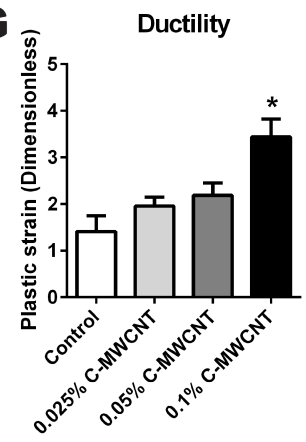

$0.05 \%$ C-MWCNT

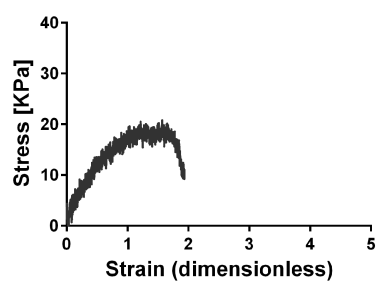

Elasticity

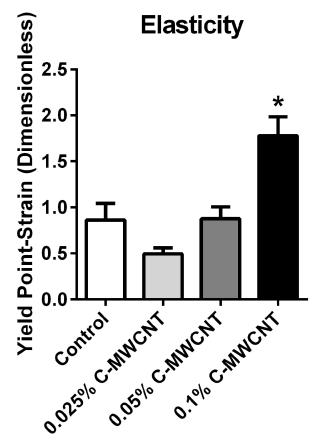

H

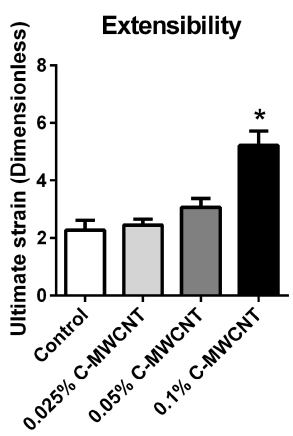

$0.1 \%$ C-MWCNT

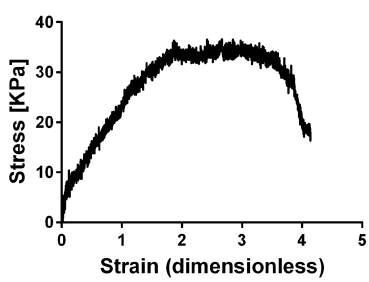

E
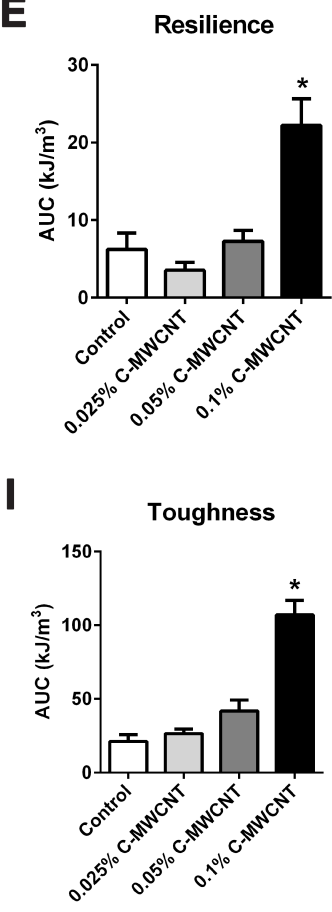

Figure 4 Effect of C-MWCNT on the mechanical properties of ECTs. Representative stress-strain curves of ECTs supplemented with increasing concentrations of C-MWCNT are depicted (A). Data as to Young's modulus [stiffness] (B), yield point-stress [proportional limit] (C), yield point strain [elasticity] (D), resilience is the area under the curve $(A \cup C)$ of the elastic phase $(\mathbf{E})$, maximum tensile stress [strength] $(\mathbf{F})$, plastic strain [ductility] (G), ultimate strain [extensibility] $(\mathbf{H})$, the total area under the stress-strain curve [toughness] (I). * $p<0.05$ compared to the corresponding control as assessed by a one-way ANOVA with Dunnett's post hoc test, $n=7-8 \mathrm{ECT} /$ group.

and decreased strain is typically found for the scarred $\operatorname{skin}^{57}$ and is a result of the structural incoherence in the scar caused by myofibroblasts. ${ }^{58}$ However, we believe that a higher content of myofibroblasts is not a feasible explanation for our result. Myofibroblasts display higher tensile forces than their fibroblast counterparts, and consequently, they give rise to more compacted and/or contracted tissues. ${ }^{56,59,60}$ ECTs with $0.025 \%$ C-MWCNT, however, did not differ in their CSAs and contraction behavior from control ECTs.

The likeliest explanation for the stiffness increase seen in the $0.025 \% \mathrm{C}$-MWCNT containing ECTs is the presence of C-MWCNT itself, as several studies have demonstrate that MWCNT enhance the Young's module of different types of non-cellular composites, ie including chitosan or collagen. Most often the increase in stiffness was found to be dependent on the concentration of the MWCNT. ${ }^{61-65}$ If this holds true for MWCNT-containing cell-populated tissues is unfortunately not clear as there is only few data available. Moreover, the investigated tissues were in most cases generated in a two-step process including the independent generation of the scaffold followed by cell seeding. ${ }^{66,67}$ We believe that seeding cells in a preadjusted 3D environment results in very different effects, than letting the cells build their own environment based on their biological specifications. Especially, fibroblasts react highly sensitive to their mechanical environment. In general, these cells adapt their intracellular tension, which is mainly conferred by 
the actin cytoskeleton, according to the experienced mechanical environment. ${ }^{59,68-70}$ By adding C-MWCNT, we expected that the fibroblasts experience a higher resistance while trying to contract the tissues, which would result in a more pronounced myofibroblastic phenotype. This idea was further supported by data demonstrating that MWCNT could directly induce the transdifferentiation of different types of fibroblasts into myofibroblasts..$^{71-73}$ However, as mentioned above neither the CSA data nor the contraction analysis of C-MWCNT containing ECTs supported this hypothesis.

Moreover, taking the stiffness-enhancing function of MWCNT and the suggested effect on fibroblast transdifferentiation into consideration, it was all the more surprising that concentrations above $0.025 \%$ of C-MWCNT lowered the stiffness again and resulted instead in the presence of $0.1 \%$ C-MWCNT in a pronounced increase in tissue elasticity and ductility, and consequently in an increased resilience and toughness. Currently we are not able to explain these findings mechanistically, but we believe that several factors could add to the observed effects.

The high concentration of C-MWCNT might have impacted the formation and cross-linking of collagen fibrils, which could result at least in part in the observed effects. In this context, it was shown that eg single-walled CNT could indeed affect the collagen fibril assembly kinetics and the collagen fibril morphology resulting in thicker and stiffer collagen fibers. ${ }^{74}$ Another important factor, which influence the viscoelastic properties of collagen fibrils is intermolecular cross-linking, ie exerted by the enzymatic family of lysyl oxidases. Inhibition of these enzymes in vivo in rats was shown to result in more ductile tail tendons compared to tendons from untreated rats. However, the inhibition of cross-linking also reduced the elastic modulus and the strength of the tendons, ${ }^{75}$ which is in line with a computational model that calculated the influence of cross-links on the viscoelastic properties of collagen fibrils. ${ }^{76}$ We hypothesize that the more than 2-fold increase in the length of the plastic phase observed in our ECT with $0.1 \%$ C-MWCNT could result from an inhibition in cross-linking. Moreover, we believe that the enhanced elasticity and strength, which is in contrast to the expectations considering a decrease in cross-linking, is a consequence of the mechanical stabilization given by the C-MWCNT.
Future studies including morphological analyses of the ECT with e.g high-resolution transmission electron microscopy could help to gain more insight in the structural organization of the single components of these tissues. Moreover, the use of other biocompatible scaffold material with a different geometry, like dispersed graphene oxide nanosheets, or a more complex composition, like nitrogen-doped MWCNT-cellulosenanohydroxyapatite composites, could help to get insights in the structural-functional relationship of ECTs. ${ }^{21,77}$ How the C-MWCNT might affect crosslinking is an open question. This could be simply due to sterical hindrance of the fibril assembly by the MWCNT or based on a more indirect effect, potentially involving the embedded cells. In a recent study, it was demonstrated that fibroblasts are able to engulf MWCNT. The consequence was a decrease in intracellular stiffness most likely due to a disaggregation of actin filaments. ${ }^{78}$ The resulting increase in G-actin could finally result in a decreased expression of lysyl oxidase. ${ }^{44}$ Further studies are needed to address the expression and activity of lysyl oxidases in the C-MWCNT-supplemented ECT.

\section{Microscopic Examination of ECTs}

To gain more insight in the orientation and morphology of the embedded cells, TRITC-Phalloidin was used to stain the actin fibers of HFF-1 in ECT sections, while DAPI was used to stain the nuclei (Figure 5). From the fluorescence images of the control ECTs, it can be noticed that the cells are aligned along their stretch axis, thus forming anisotropic tissues (Figure 5A-C). The applied C-MWCNT did not hamper the alignment of the embedded cells at any concentration and the C-MWCNT were evenly distributed within the tissues (Figure 5D-L). That carbon nanotubes do not disturb or even improve cell alignment has been already shown in collagen-based cardiac constructs. ${ }^{55}$ Whether or not the actin cytoskeleton is changed in the ECT with C-MWCNT is difficult to assess by fluorescence microscopy due to the opaque nature of the carbon nanotubes. As mentioned above, other optical methods, like high-resolution transmission electron microscopy, could help to answer this question.

In summary, our data demonstrate that $0.025 \%$ of C-MWCNT moderately, but significantly increased the stiffness of ECTs, whereas $0.1 \%$ of C-MWCNT impaired tissue contraction, and increased the elasticity, extensibility, and the ability of energy absorption. Thus, 

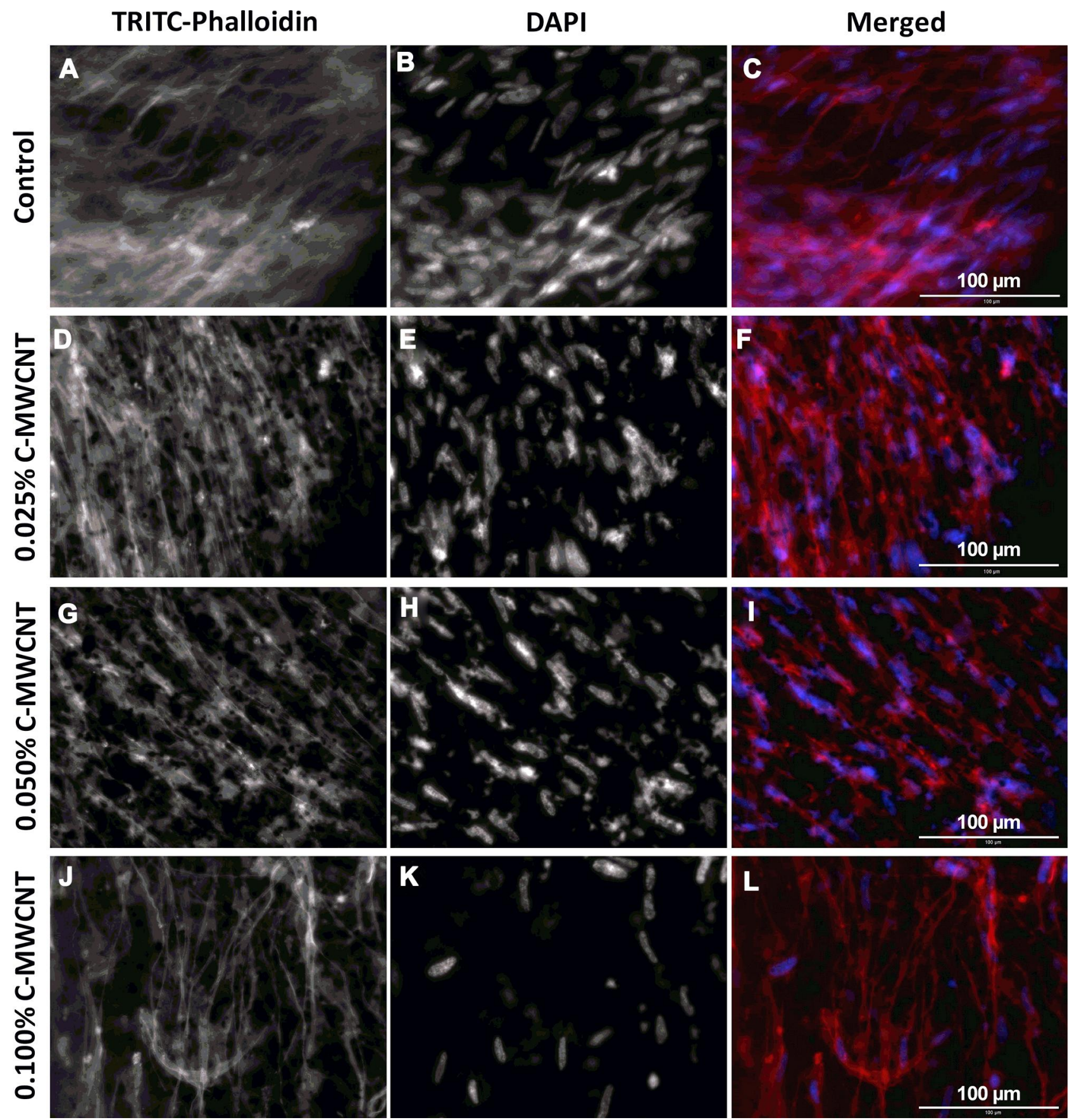

Figure 5 Effect of C-MWCNT on actin fiber formation in ECT. (A, D, G and J) actin cytoskeleton of the fibroblasts stained with TRITC-phalloidin (red); (B, E, H and K) nuclei stained with DAPI (blue); (C, F, I and L) merged images from DAPI and TRITC-phalloidin. In all conditions the HFF-I cells were aligned in parallel to the long side of the tissues.

our data demonstrates that the outcome of the application of C-MWCNT is strongly dependent on the added concentration and do not simply follow a linear relationship.

\section{Conclusion}

The incorporation of $0.1 \%$ C-MWCNT in ECTs could favorably improve their biomechanical properties, which could be utilized for potential in vivo applications in connective tissue repair, like the dermis layer in the skin, tendons and ligaments, which require high endurance capacities against external stressors.

\section{Funding}

NK and MA: The project was kindly funded and supported by the Palestinian Ministry of Higher Education (fund ID 
number ANNU-MoHE-1819-Sc010), the Deanship of Scientific Research at An-Najah National University (ANNU-1920-Sc016) and by the German Academic Exchange Service (DAAD). This work was further funded by the German Research Foundation (DFG) by the project IRTG 1816 to GLS. AR was funded by the Higher Education Commission of Pakistan. SL is supported by the German Center for Cardiovascular Research (DZHK). W.H.Z is supported by the German Research Foundation (DFG; CRC 1002 C04 and S01, IRTG 1816), the German Center for Cardiovascular Research (DZHK), and the Leducq Foundation.

\section{Disclosure}

W.H.Z. is co-inventor of the applied multiwell-plate culture format. He is a founder and shareholder of Myriamed $\mathrm{GmbH}$. The authors report no other potential conflicts of interest for this work.

\section{References}

1. Christ GJ, Saul JM, Furth ME, Andersson KE, Nader MA. The pharmacology of regenerative medicine. Pharmacol Rev. 2013;65 (3):1091-1133. doi:10.1124/pr.112.007393

2. Mao AS, Mooney DJ. Regenerative medicine: current therapies and future directions. Proc Natl Acad Sci $U$ S A. 2015;112 (47):14452-14459. doi:10.1073/pnas.1508520112

3. Dieckmann C, Renner R, Milkova L, Simon JC. Regenerative medicine in dermatology: biomaterials, tissue engineering, stem cells, gene transfer and beyond. Exp Dermatol. 2010;19(8):697-706. doi:10.1111/j.1600-0625.2010.01087.x

4. Jacques E, Suuronen EJ. The progression of regenerative medicine and its impact on therapy translation. Clin Transl Sci. 2020;13 (3):440-450. doi:10.1111/cts.12736

5. Kamrani P, Jan A. Anatomy, Connective Tissue. Treasure Island (FL): StatPearls; 2019.

6. Halper J, Kjaer M. Basic components of connective tissues and extracellular matrix: elastin, fibrillin, fibulins, fibrinogen, fibronectin, laminin, tenascins and thrombospondins. Adv Exp Med Biol. 2014;802:31-47.

7. Ushiki T. Collagen fibers, reticular fibers and elastic fibers. A comprehensive understanding from a morphological viewpoint. Arch Histol Cytol. 2002;65(2):109-126. doi:10.1679/aohc.65.109

8. Factor SM, Robinson TF. Comparative connective tissue structure-function relationships in biologic pumps. Lab Invest. 1988;58(2):150-156.

9. El Haj AJ, Hampson K, Gogniat G. Bioreactors for connective tissue engineering: design and monitoring innovations. Adv Biochem Eng Biotechnol. 2009;112:81-93. doi:10.1007/978-3-540-69357-4_4

10. Croisier FJ, Jérôme C. Chitosan-based biomaterials for tissue engineering. Eur Polym J. 2013;49(4):780-792. doi:10.1016/j. eurpolymj.2012.12.009

11. Collins PG, Avouris P. Nanotubes for electronics. Sci Am. 2000;283 (6):62-69. doi:10.1038/scientificamerican1200-62

12. Yu M-F, Files BS, Arepalli S, Ruoff RS. Tensile loading of ropes of single wall carbon nanotubes and their mechanical properties. Phys Rev Lett. 2000;84(24):5552. doi:10.1103/PhysRevLett.84.5552
13. Meo M, Rossi M. Prediction of young's modulus of single wall carbon nanotubes by molecular-mechanics based finite element modelling. Compos Sci Technol. 2006;66(11-12):1597-1605. doi:10.10 16/j.compscitech.2005.11.015

14. Harris PJ. Carbon Nanotubes and Related Structures. Cambridge: Cambridge University Press; 1991.

15. Dresselhaus MS, Dresselhaus G, Eklund PC. Science of Fullerenes and Carbon Nanotubes: Their Properties and Applications. Academic press; 1996.

16. Iijima S. Helical microtubules of graphitic carbon. Nature. 1991;354 (6348):56-58. doi:10.1038/354056a0

17. Cheng L-C, Jiang X, Wang J, Chen C, Liu R-S. Nano-bio effects: interaction of nanomaterials with cells. Nanoscale. 2013;5(9):3547. doi:10.1039/c3nr34276j

18. Baughman RH. Carbon nanotubes-the route toward applications. Science. 2002;297(5582):787-792. doi:10.1126/science.1060928

19. Lalwani G, Gopalan A, D’Agati M, et al. Porous three-dimensional carbon nanotube scaffolds for tissue engineering. J Biomed Mater Res A. 2015;103(10):3212-3225. doi:10.1002/jbm.a.35449

20. Kittana N, Assali M, Abu-Rass H, et al. Enhancement of wound healing by single-wall/multi-wall carbon nanotubes complexed with chitosan. Int J Nanomedicine. 2018;13:7195-7206. doi:10.2147/IJN. S183342

21. Zhang X, Yin X, Luo J, et al. Novel hierarchical nitrogen-doped multiwalled carbon nanotubes/cellulose/nanohydroxyapatite nanocomposite as an osteoinductive scaffold for enhancing bone regeneration. ACS Biomater Sci Eng. 2019;5(1):294-307. doi:10.1021/acsbiomaterials. 8 b00908

22. Kolanthai E, Sindu PA, Khajuria DK, et al. Graphene oxide - a tool for the preparation of chemically crosslinking free alginate-chitosancollagen scaffolds for bone tissue engineering. ACS Appl Mater Interfaces. 2018;10(15):12441-12452. doi:10.1021/acsami.8b00699

23. Dai C, Li Y, Pan W, et al. Three-dimensional high-porosity chitosan/ honeycomb porous carbon/hydroxyapatite scaffold with enhanced osteoinductivity for bone regeneration. ACS Biomater Sci Eng. 2020;6(1):575-586. doi:10.1021/acsbiomaterials.9b01381

24. Schlick SF, Spreckelsen F, Tiburcy M, et al. Agonistic and antagonistic roles of fibroblasts and cardiomyocytes on viscoelastic stiffening of engineered human myocardium. Prog Biophys Mol Biol. 2019;144:51-60. doi:10.1016/j.pbiomolbio.2018.11.011

25. Tiburcy M, Meyer T, Soong PL, Zimmermann WH. Collagen-based engineered heart muscle. Methods Mol Biol. 2014;1181:167-176.

26. Gabbiani G, Ryan GB, Majne G. Presence of modified fibroblasts in granulation tissue and their possible role in wound contraction. Experientia. 1971;27(5):549-550. doi:10.1007/BF02147594

27. Bochaton-Piallat ML, Gabbiani G, Hinz B. The myofibroblast in wound healing and fibrosis: answered and unanswered questions. F1000Res. 2016;5:752. doi:10.12688/f1000research.8190.1

28. Maione AG, Smith A, Kashpur O, et al. Altered ECM deposition by diabetic foot ulcer-derived fibroblasts implicates fibronectin in chronic wound repair. Wound Repair Regen. 2016;24(4):630-643. doi:10.1111/wrr.12437

29. Tobalem M, Levigne D, Modarressi A, et al. Hyperglycemia interacts with ischemia in a synergistic way on wound repair and myofibroblast differentiation. Plast Reconstr Surg Glob Open. 2015;3(7):e471. doi:10.1097/GOX.0000000000000443

30. Fleck CA, Simman R. Modern collagen wound dressings: function and purpose. J Am Col Certif Wound Spec. 2010;2(3):50-54. doi:10.1016/j.jcws.2010.12.003

31. Pilcher BK, Dumin JA, Sudbeck BD, Krane SM, Welgus HG, Parks WC. The activity of collagenase-1 is required for keratinocyte migration on a type I collagen matrix. J Cell Biol. 1997;137 (6):1445-1457. doi:10.1083/jcb.137.6.1445

32. Yang W, Sherman VR, Gludovatz B, et al. On the tear resistance of skin. Nat Commun. 2015;6(1):6649. doi:10.1038/ncomms7649 
33. Debels H, Hamdi M, Abberton K, Morrison W. Dermal matrices and bioengineered skin substitutes: a critical review of current options. Plast Reconstr Surg Glob Open. 2015;3(1):e284. doi:10.1097/ GOX.0000000000000219

34. Rao CNR, Satishkumar BC, Govindaraj A, Nath M. Nanotubes. ChemPhysChem. 2001;2(2):78-105.

35. Gutsche CS, Reich C, Thomsen J. Maultzsch: carbon nanotubes, basic concepts and physical properties. Colloid Polym Sci. 2004;282(11):1299. doi:10.1007/s00396-004-1180-6

36. Liu Z, Robinson JT, Tabakman SM, Yang K, Dai H. Carbon materials for drug delivery \& cancer therapy. Mater Today. 2011;14 (7):316-323. doi:10.1016/S1369-7021(11)70161-4

37. Ma L, Gao C, Mao Z, et al. Collagen/chitosan porous scaffolds with improved biostability for skin tissue engineering. Biomaterials. 2003;24(26):4833-4841. doi:10.1016/S01429612(03)00374-0

38. Han CM, Zhang LP, Sun JZ, Shi HF, Zhou J, Gao CY. Application of collagen-chitosan/fibrin glue asymmetric scaffolds in skin tissue engineering. $J$ Zhejiang Univ Sci B. 2010;11(7):524-530. doi:10.1631/jzus.B0900400

39. Hosseinnejad M, Jafari SM. Evaluation of different factors affecting antimicrobial properties of chitosan. Int $J$ Biol Macromol. 2016;85:467-475. doi:10.1016/j.ijbiomac.2016.01.022

40. Alburquenque C, Bucarey SA, Neira-Carrillo A, Urzua B, Hermosilla G, Tapia CV. Antifungal activity of low molecular weight chitosan against clinical isolates of Candida spp. Med Mycol. 2010;48 (8):1018-1023. doi:10.3109/13693786.2010.486412

41. Matica MA, Aachmann FL, Tondervik A, Sletta H, Ostafe V. Chitosan as a wound dressing starting material: antimicrobial properties and mode of action. Int J Mol Sci. 2019;20(23):5889. doi:10.3390/ijms20235889

42. Jatho A, Hartmann S, Kittana N, et al. RhoA ambivalently controls prominent myofibroblast characteritics by involving distinct signaling routes. PLoS One. 2015;10(10):e0137519. doi:10.1371/journal. pone. 0137519

43. Ongherth A, Pasch S, Wuertz CM, et al. p63RhoGEF regulates autoand paracrine signaling in cardiac fibroblasts. $\mathrm{J} \mathrm{Mol} \mathrm{Cell} \mathrm{Cardiol.}$ 2015;88:39-54. doi:10.1016/j.yjmcc.2015.09.009

44. Santos GL, Hartmann S, Zimmermann W-H, Ridley A, Lutz S. Inhibition of Rho-associated kinases suppresses cardiac myofibroblast function in engineered connective and heart muscle tissues J Mol Cell Cardiol. 2019;134:13-28. doi:10.1016/j.yjmcc.20 19.06.015

45. Dworatzek E, Mahmoodzadeh S, Schriever C, et al. Sex-specific regulation of collagen I and III expression by 17beta-Estradiol in cardiac fibroblasts: role of estrogen receptors. Cardiovasc Res. 2019;115(2):315-327. doi:10.1093/cvr/cvy185

46. Vettel C, Lammle S, Ewens S, et al. PDE2-mediated cAMP hydrolysis accelerates cardiac fibroblast to myofibroblast conversion and is antagonized by exogenous activation of cGMP signaling pathways. Am J Physiol Heart Circ Physiol. 2014;306(8):H1246-1252. doi:10.1152/ajpheart.00852.2013

47. Driesen RB, Nagaraju CK, Abi-Char J, et al. Reversible and irreversible differentiation of cardiac fibroblasts. Cardiovasc Res. 2014;101 (3):411-422. doi: $10.1093 / \mathrm{cvr} / \mathrm{cvt} 338$

48. Wille JJ, Elson EL, Okamoto RJ. Cellular and matrix mechanics of bioartificial tissues during continuous cyclic stretch. Ann Biomed Eng. 2006;34(11):1678-1690. doi:10.1007/s10439-0069153-1

49. Ngu JM, Teng G, Meijndert HC, et al. Human cardiac fibroblast extracellular matrix remodeling: dual effects of tissue inhibitor of metalloproteinase-2. Cardiovasc Pathol. 2014;23(6):335-343. doi:10.1016/j.carpath.2014.06.003

50. Sadeghi AH, Shin SR, Deddens JC, et al. Engineered 3D cardiac fibrotic tissue to study fibrotic remodeling. Adv Healthc Mater. 2017;6(11).
51. Wakatsuki T, Kolodney MS, Zahalak GI, Elson EL. Cell mechanics studied by a reconstituted model tissue. Biophys J. 2000;79 (5):2353-2368. doi:10.1016/S0006-3495(00)76481-2

52. Elson EL, Genin GM. Tissue constructs: platforms for basic research and drug discovery. Interface Focus. 2016;6(1):20150095. doi:10.1098/rsfs.2015.0095

53. MacDonald RA, Voge CM, Kariolis M, Stegemann JP. Carbon nanotubes increase the electrical conductivity of fibroblast-seeded collagen hydrogels. Acta Biomater. 2008;4(6):1583-1592. doi:10.1016/ j.actbio.2008.07.005

54. Tosun Z, McFetridge PS. A composite SWNT-collagen matrix: characterization and preliminary assessment as a conductive peripheral nerve regeneration matrix. $J$ Neural Eng. 2010;7(6):066002. doi:10.1088/1741-2560/7/6/066002

55. Sun H, Zhou J, Huang Z, et al. Carbon nanotube-incorporated collagen hydrogels improve cell alignment and the performance of cardiac constructs. Int $J$ Nanomedicine. 2017;12:3109-3120. doi:10.2147/IJN.S128030

56. Doan KT, Kshetri P, Attamakulsri N, et al. The effect of chitosan derivatives on the compaction and tension generation of the fibroblast-populated collagen matrix. Molecules. 2019;24(15):2713. doi:10.3390/molecules24152713

57. Clark JA, Cheng JC, Leung KS. Mechanical properties of normal skin and hypertrophic scars. Burns. 1996;22(6):443-446. doi:10.1016/0305-4179(96)00038-1

58. Wolfram D, Tzankov A, Pulzl P, Piza-Katzer H. Hypertrophic scars and keloids-a review of their pathophysiology, risk factors, and therapeutic management. Dermatol Surg. 2009;35(2):171-181. doi:10.1111/j.1524-4725.2008.34406.x

59. Kollmannsberger P, Bidan CM, Dunlop JWC, Fratzl P, Vogel V. Tensile forces drive a reversible fibroblast-to-myofibroblast transition during tissue growth in engineered clefts. Sci Adv. 2018;4(1): eaao4881. doi:10.1126/sciadv.aao4881

60. Spiekman M, Przybyt E, Plantinga JA, Gibbs S, van der Lei B, Harmsen MC. Adipose tissue-derived stromal cells inhibit TGF-beta1-induced differentiation of human dermal fibroblasts and keloid scar-derived fibroblasts in a paracrine fashion. Plast Reconstr Surg. 2014;134(4):699-712. doi:10.1097/PRS.0000000000000504

61. Arslantunali D, Budak G, Hasirci V. Multiwalled CNT-pHEMA composite conduit for peripheral nerve repair. J Biomed Mater Res A. 2014;102(3):828-841. doi:10.1002/jbm.a.34727

62. Wang SF, Shen L, Zhang WD, Tong YJ. Preparation and mechanical properties of chitosan/carbon nanotubes composites. Biomacromolecules. 2005;6(6):3067-3072. doi:10.1021/bm050378v

63. Aryaei A, Jayatissa AH, Jayasuriya AC. Mechanical and biological properties of chitosan/carbon nanotube nanocomposite films. J Biomed Mater Res A. 2014;102(8):2704-2712. doi:10.1002/jbm. a.34942

64. Kroustalli A, Zisimopoulou AE, Koch S, et al. Carbon nanotubes reinforced chitosan films: mechanical properties and cell response of a novel biomaterial for cardiovascular tissue engineering. J Mater Sci Mater Med. 2013;24(12):2889-2896. doi:10.1007/s10856-013-5029-8

65. Baktur R, Yoon S-H, Kwon S. Effects of multiwalled carbon nanotube reinforced collagen scaffolds on the osteogenic differentiation of mesenchymal stem cells. J Nanomater. 2013;2013:904083. doi:10.1155/2013/904083

66. Ho CM, Mishra A, Lin PT, et al. 3D printed polycaprolactone carbon nanotube composite scaffolds for cardiac tissue engineering. Macromol Biosci. 2017;17(4):1600250. doi:10.1002/mabi.201600250

67. Khalid P, Hussain MA, Rekha PD, Arun AB. Carbon nanotube-reinforced hydroxyapatite composite and their interaction with human osteoblast in vitro. Hum Exp Toxicol. 2015;34 (5):548-556. doi:10.1177/0960327114550883

68. Squier CA. The effect of stretching on formation of myofibroblasts in mouse skin. Cell Tissue Res. 1981;220(2):325-335. doi:10.1007/ BF00210512 
69. Yeung T, Georges PC, Flanagan LA, et al. Effects of substrate stiffness on cell morphology, cytoskeletal structure, and adhesion. Cell Motil Cytoskeleton. 2005;60(1):24-34. doi:10.1002/cm.20041

70. Walcott S, Sun SX. A mechanical model of actin stress fiber formation and substrate elasticity sensing in adherent cells. Proc Natl Acad Sci U S A. 2010;107(17):7757-7762. doi:10.1073/pnas.0912739107

71. Hindman B, Ma Q. Carbon nanotubes and crystalline silica induce matrix remodeling and contraction by stimulating myofibroblast transformation in a three-dimensional culture of human pulmonary fibroblasts: role of dimension and rigidity. Arch Toxicol. 2018;92 (11):3291-3305. doi:10.1007/s00204-018-2306-9

72. He X, Young SH, Schwegler-Berry D, Chisholm WP, Fernback JE, Ma Q. Multiwalled carbon nanotubes induce a fibrogenic response by stimulating reactive oxygen species production, activating NF-kappaB signaling, and promoting fibroblast-to-myofibroblast transformation. Chem Res Toxicol. 2011;24(12):2237-2248. doi:10.1021/tx200351d

73. Wang P, Wang Y, Nie X, Braini C, Bai R, Chen C. Multiwall carbon nanotubes directly promote fibroblast-myofibroblast and epithelial-mesenchymal transitions through the activation of the TGF-beta/Smad signaling pathway. Small. 2015;11(4):446-455. doi:10.1002/smll.201303588
74. Kim T, Sridharan I, Zhu B, Orgel J, Wang R. Effect of CNT on collagen fiber structure, stiffness assembly kinetics and stem cell differentiation. Mater Sci Eng C Mater Biol Appl. 2015;49:281-289. doi:10.1016/j.msec.2015.01.014

75. Puxkandl R, Zizak I, Paris O, et al. Viscoelastic properties of collagen: synchrotron radiation investigations and structural model. Philos Trans R Soc Lond B Biol Sci. 2002;357(1418):191-197. doi:10.1098/rstb.2001.1033

76. Depalle B, Qin Z, Shefelbine SJ, Buehler MJ. Influence of cross-link structure, density and mechanical properties in the mesoscale deformation mechanisms of collagen fibrils. J Mech Behav Biomed Mater. 2015;52:1-13. doi:10.1016/j.jmbbm.2014.07.008

77. Yang W, Zhong Y, He C, et al. Electrostatic self-assembly of pFe3O4 nanoparticles on graphene oxide: a co-dispersed nanosystem reinforces PLLA scaffolds. J Adv Res. 2020;24:191-203. doi:10.1016/j. jare.2020.04.009

78. Pastrana HF, Cartagena-Rivera AX, Raman A, Avila A. Evaluation of the elastic Young's modulus and cytotoxicity variations in fibroblasts exposed to carbon-based nanomaterials. J Nanobiotechnology. 2019;17(1):32. doi:10.1186/s12951-019-0460-8
International Journal of Nanomedicine

\section{Publish your work in this journal}

The International Journal of Nanomedicine is an international, peerreviewed journal focusing on the application of nanotechnology in diagnostics, therapeutics, and drug delivery systems throughout the biomedical field. This journal is indexed on PubMed Central, MedLine, CAS, SciSearch ${ }^{\mathbb{}}$, Current Contents ${ }^{\mathbb{R}} /$ Clinical Medicine,

\section{Dovepress}

Journal Citation Reports/Science Edition, EMBase, Scopus and the Elsevier Bibliographic databases. The manuscript management system is completely online and includes a very quick and fair peer-review system, which is all easy to use. Visit http://www.dovepress.com/ testimonials.php to read real quotes from published authors. 